\title{
Síndromos funcionales cardíacos y estados ansiosos del embarazo tratados con Clorpromazine (1)
}

\author{
Profesor Dino Cazzola \\ Adjunto y Docente Libre de la Clínica Obstétrica y Ginecológica de la \\ Universidad de Pavía (Italia). \\ (Artículo enviado directamente para su publicación en la Revista de la \\ Sociedad Colombiana de Obstetricia y Ginecología. Traducción de la Direc- \\ ción (G. L. E.).
}

Las observaciones personales que nos proponemos referir tienen de nuevo un interés olvidado. Durante los últimos tiempos se ha hablado mucho de estas manifestaciones funcionales, particularmente en io que concierne a nuestra especialidad. Primitivamente restringidas al terreno de la patología menstrual y preclimatérica, han llegado a ser también materia de estudio en el campo de la patoiog'a gravídica.

El estado neuro-hormonal tiene gran influencia en ia génesis de tales manifestaciones, y la mujer durante las grandes y pequeñas crisis sexuales muestra una estallante labilidad en este sistema.

Hace algunos años Lenegre y Coblentz ilustraron y documen_ taron de modo claro y convincente un síndrome cardíaco rebel_ de concomitante con la menstruación en mujeres que no presen. taban cardiopatía orgánica, caracterizado por palpitaciones y aun edema pulmonar agudo febril. Según taies autores esto debería atribuírse al hiperfoliculinismo. La explicación del mecanismo a través del cual la foliculina obraría es la siguiente: la hormona provoca un aumento de la retención hídrica, con la consiguiente descompensación mecánica y un desequilibrio vago_ simpático que lleva, en el aparato cardio-circulatorio, a ios conocidos resultados derivados de las alteraciones del sistema auto-

(1) Nombre químico del Largactil. 
mático. No es de dudar que, en parte, éste sea el mecanismo acer. tado; pero que toda la compieja sintomatología sea atribuíble a él. hace nacer una justificada reserva. Basta pensar que la cues. tión del hiperfoliculinismo puro como causa primaria y exclusiva de enfermedad, va perdiendo su valor en frente a la noción verdaderamente sugestiva de la receptividad y reactividad de ios diversos tejidos. En conformidad a su tesis Lenegre y Coblentz nan propuesto en estos casos la administración de medicinas sedantes junto con acetato de testosterona.

El conjunto de síndromos card acos en mujeres no cardiópa_ tas reclama por evidente afinidad las crisis ansiosas do observación no rara en el premenstruo, la menopausia y también en ei embarazo.

Durante los úitimos años se han hecho progresos acerca de la génesis de estas complejas manifestaciones (Gammon y Churchill; Schlesinger, Hunter, Waterfall, Jacobson, etc.) y la orientación terapéutica se ha ido uniformando de acuerdo con tales recientes interpretaciones.

Mientras que se puede individualizar la razón por ia cual la mujer está ansiosa y preocupada no se puede fácilmente precisar cuáles procesos funcionales pueden estar envueltos en la realización de este sstado.

Dentro de las interpretaciones propuestas merece atención la de Dixon y coll.: la ansiedad sería la expresión emotiva de un estado fisiológico en el cual el organismo, considerado en conjunto, se prepara a combatir una amenaza ambiental cualquie_ ra; los prodromos etarían caracterizados por un conjunto de síntomas premonitores en los que participaría todo el sistema neurovascular, se tendría, en otras palabras, lo que Jacobsen llama "Hipertensión neuro-muscuiar".

La ansiedad representaría allí uno de los síntomas de un estado generalizado de hipertensión neuro_muscuiar. Los estados de tensión ansiosa. que según Dixon y col. son fenómenos condicionados, podrían ser corregidos o curados reeducando o llevando estos sujetos a un estado psicológico que los haga sobrellevar confortablemente. La técnica de la reiajación de Jacobson podra obtenerse empleando los neuroplégicos, los que por su conocida acción biológica responden a tal fin. De aquí la justificación de los que hasta ahora han empleado ia clorpromazine, en casos del género a que nos referimos, para conseguir las condiciones anotadas. 
Permítasenos ahora, para no continuar con el mecanismo patogénico, señalar algunos conocimientos farmaco_biológicos que se tienen sobre li: Clorpromazine, preparado que mejor que ctros neuropléjicos, presenta características taies que le permiten ser empleado en contingencias similares.

Su propiedad simpaticolítica y antiespástica le permite ejercer un efecto antifibrilatorio. Inyectado endovenosamente, atenúa en el conejo la irregularidad del ritmo provocado por la aconitina suministrada endovenosamente e impide el bloqueo atrioventricular determinado por el ácido adenosinfosfórico. El efecto vasodilatador, puesto en evidencia sobre el corazón del cone_ jo aislado y perfundido, concurre a aumentar el rendimiento de la arteria coronaria. Es igualmente de anotar que la inyección de Clorpromazine por vía subcutánea protege al ratón del edema de ovoalbúmina. Estos fenómenos se deben a ia capacidad del fármaco de inhibir la permeabilidad de los capilares.

En la distonia neuro vegetativa, especialmente cuando se irata de una distonia simple que repercute sobre un solo órgano o aparato, el éxito obtenido con tai neuroplégico está ampliamente reconocido. Entran en esta categoría ciertos disturbios cardiacos. como la angina de pecho, el infarto del miocardio, que, al menos en parte, dependen de un desequilibrio neuro-vegetativo. Ellos se benefician del empleo dei preparado aun cuando han fallado las terapéuticas del uso común.

En apoyo de cuanto puede ser útil en general, ei empleo de los neuroplégicos en los disturbios de la funcionalidad cardíaca, recordemos lo que han afirmado Dubouchet, Latscha y Passalecq acerca de los favorabies efectos obtenidos en casos que presenta. han alteraciones de la conducción nerviosa.

Para la terapéutica del estado ansioso asociado al menos a trastornos cardíacos funcionales, se han propuesto métodos diversos y fármacos diferentes; entre éstos ha prevalecido el criterio de emplear vasodilatadores, aunque no siempre han llevado a resultados positivos. La reconocida (Bonnet y otros) propiedad espec fica de los neuroplégicos, en lo que se refiere ya ai bloqueo de los ganglios autónomos con inhibición de los impulsos simpáticos vasoconstrictores, ya a la actividad adrenolítica o simpaticolitica de efecto local, lo ha hecho preferir.

Entre los numerosos neuroplégicos hemos dado preferencia a la Clorpromazine, porque pensamos que mejor que otros responde a las exigencias especificas del sindromo que tratamos. 
Además en manifestaciones cardiacas ligadas a desequilibrio neuro_vegetativo (Sigwald y Bouttier, Coutchier, Delay. Deniker, Harl., Cahn, Dubrasquet y Melon, etc.) han empleado con buenos resultados este preparado. Experiencias han sido hechas también en los estados ansiosos por Delay, Deschamp y coll., Massari, Belloni, Baruk, Launay y Lelord.

No creemos aprobado, por lo menos en este momento, que sea necesario hacer relación de toda la casuistica que ha recibido este tratamiento en ias manifestaciones citadas aparecidas en el embarazo. Sin embargo, por ciertas observaciones personales de las cuales ya se ha hablado en otro lugar. por las afirmaciones de Lenegre y Coblentz, reafirmadas por Lutembacher y Gaiimard, hemos pensado que no estaría privado de interés el señalar aquellos casos de embarazo que aurante el periodo de la gestación sufrieron tales disturbios y mejoraron o curaron con el tratamiento de la Clorpromazine.

\section{CASUISTICA}

I.-G. Carla, veinticuatro años, habitante de Campomorfo (Pavía).

No ced: 4.147 ; $\mathrm{N}^{\circ}$ reg.: 315.

Diagnóstico: embarazo en el curso del noveno mes, primípara. Desde hace cerca de un mes la paciente, preocupada con el avecinamiento del parto, se muestra fácilmente irritable por la menor contrariedad en su trabajo cuotidiano y nota una agitación continua que, a más de incapacitarla, le disminuye las horas de sueño.

El tratamiento con Clorpromazine hecho en la clínica hace desaparecer en poco tiempo tal sintomatología.

II.-B. Linda, treinta años, habitante de Belgioso (Pavía).

$\mathrm{N}^{\circ}$ ced: 3.660 ; $\mathrm{N}^{\circ}$ reg.: 231.

Diagnóstico: embarazo en el noveno mes, primípara. Después de los primeros meses de gestación la primípara se muestra fácilmente impresionable por motivos baladíes y siente cardiospasmo y disnea con ocasión de estas crisis. Viene convaleciente como cardiópata. El examen clínico resultó negativo.

El tratamiento con Clorpromazine durante 20 días hace desaparecer el síndromo antes del parto.

III.-G. Ida, treinta y tres años, aldeana de Vidigulfo (Pavía).

$\mathrm{N}^{\circ}$ ced.: 3.330 . $\mathrm{N}^{\text {o }}$ reg.: 214.

Diagnóstico: trabajo de parto en tercípara.

La paciente llega a la clínica presa de profunda agitación. El tratamiento con la Clorpromazine lleva a la calma en breve tiempo a la paciente.

IV.-C. María, veinticuatro años, habitante de Pavía.

No ced.: 631; No $^{\text {reg.: }} 39$.

Diagnóstico: trabajo de parto en primípara. 
La paciente llega a la clínica quejándose de cardiospasmo y dificultad de respirar, sensación de nudo en la garganta.

El examen cardiológico es negativo. No se aprecia aumento de tamaño del tiroides. La terapéutica mejora sensiblemente este estado.

V.-C. María, cuarenta y cuatro años, habitante de Pavía.

$\mathrm{N}^{\text {o }}$ ced.: 5.267 ; $\mathrm{N}^{\circ}$ reg.: 323.

Diagnóstico: embarazo en el curso del octavo mes. Secundípara.

Desde hace algunos meses la paciente presenta un estado típicamente ansioso, es impulsiva y hasta violenta.

Después de 20 días de tratamiento retorna a la tranquilidad y su gestación continúa perfectamente controlada.

VI.-P. Gianna, veinticinco años, habitante de Sannazaro (Pavía).

$\mathrm{N}^{\circ}$ ced.: 14.853 ; $\mathrm{N}^{\circ}$ reg.: 913.

Diagnóstico: embarazo en el curso del noveno mes, secundípara.

La paciente está atormentada por sensaciones de nudo en la garganta que hacen difícil la deglución, se muestra irritable al menor obstáculo. No s:e aprecia aumentot del volumen tiroideo.

Después de 20 días de tratamiento el síndromo desaparece.

VII.-T. Vicenza, treinta y nueve años, habitante de Graffignana (Milán). $\mathrm{N}^{\circ}$ ced.: 4.463 ; $\mathrm{N}^{\circ}$ reg.: 312 .

Diagnóstico: embarazo en el curso del séptimo mes, tercípara.

La paciente llega a la clínica agitada, taquicárdica, tiene impulsos incontrolables. Mejoramiento después de 25 días de tratamiento.

VIII.-L. Angela, veintisiete años, habitante de Vidigu!fo (Pavía).

$\mathrm{N}^{0}$ ced.: $11.938 ; \mathrm{N}^{\circ}$ reg.: 740 .

Diagnóstico: embarazo en el curso del octavo mes, primípara.

De algunos meses acá la han sobrecogido crisis de profunda angustia que la hacen impulsiva e incoherente.

Mejoramiento después de 20 días de tratamiento.

IX.-V. Teresa, treinta años, residente en Vidigulfo (Pavía).

$\mathrm{N}^{\circ}$ ced.: 3.652 ; $\mathrm{N}^{\circ}$ reg.: 244.

Diagnóstico: embarazo en el curso del noveno mes, secundípara.

La paciente entra como cardiópata. El examen clínico es negativo.

La disnea y la taquicardia de que se quejaba desaparecieron fácilmente después de breve tiempo de tratamiento.

X.-P. Elsa, veintiocho años, habitante de Siziano (Pavía).

$\mathrm{N}^{\circ}$ ced.: 3.019 ; $\mathrm{N}^{\circ}$ reg.: 200.

Diagnóstico: embarazo en el tercer mes de gestación, primípara.

La paciente sufre cada dos o tres días de crisis de angustia que la hacen insomne. Mejoría después de 20 días de tratamiento.

XI.-L. Clara, veinte años, habitante de Casanova (Pavía).

$\mathrm{N}^{\circ}$ ced.: 1.965 ; $\mathrm{N}^{\circ}$ reg.: 125.

Diagnóstico: embarazo en el curso del noveno mes, primípara.

La paciente entra a la clínica preocupada con su estado. Fácilmente irritable, acusa hostilidad al medio que la rodea y acusa disturbios imaginarios. Mejoramiento a los pocos días del tratamiento. 


\section{Consideraciones y conclusiones}

A mi parecer ías observaciones presentadas merecen un comentario, porque a más del resultado terapéutico, algunas particularidades se prestan a interesantes consideraciones en cuan. to a las relaciones causales entre el estado gravídico y las manifestaciones extragenitales.

Ei primer aspecto de la cuestión, ei que tiene mayor valor desde el punto de vista práctico, se refiere al éxito terapéutico. Datos objetivos y subjetivos (estos últimos tienen un significado superior a los primeros) prueban que el empleo de la Clorpromazine lleva. ya en el caso de disturbios funcionales, ya en el estado ansioso, ya cuando se combinan los dos fenómenos, a resultados positivos. El efecto puede ser de grado tan notorio que se podría hablar directamente en algunos casos, de curación.

La duración de la acción, en relación con los otros fármacos comunmente usados, es sin duda mayor. No solo sino que a diferencia de los sedativos en general, se asiste desde el per odo de tratamiento a una consolidación del resultado obtenido.

Esto hace pensar que no se trata de un medicamento que se limite a bloquear los efectos últimos de una causa primitiva sin interferir de ninguna manera sobre aqueilos que son presumiblemente los mecanismos de realización. sino de un medicamento que, operando en profundidad, interrumpe la concatenación de los eventos a un nivel más bajo, aunque no se llegue en el sen. tido estricto a una remoción de la causa primitiva.

Además de la opinión compartida por muchos de que en el ori_ gen de éstas y de otras manifestaciones de naturaleza funcional extragenital existe un exceso de alguna hormona, en particular de la foliculina, orientaría en este sentido. Se recuerda a este respecto cuanto han comprobado Miller, Waever y otros desde ei empleo de grandes cantidades de hormonas (esteroides) en casos de tratamiento erradamente impuestos.

Los disturbios cardíacos en consideración, se encuentran, se_ gún Lenegre y Coblentz, con mayor frecuencia en sujetos de feminidad destacada, de tipo sutil y grácil, en las que las reglas son abundantes y frecuentes, que presentan turgencia premenstrual de ios senos, falsas reglas y tensión abdominal premenstrual. Y añadamos: "la foliculina agrava el síndromo menstrual de la estenosis mitral y así también el embarazo que correspon- 
de a una inundación masiva de foliculina". Es necesario además tener en cuenta las otras hormonas: la progesterona y las hor. monas hipofisiarias (ganadotropinas y posthipofisiarias).

Por otra parte Baruk. David, Racine y Lauret han comprobado vasodilatación muy apreciable de los grandes y pequeños vasos cerebrales bajo la influencia de la foliculina; la biopsia cerebral ha demostrado que ella determina dilatación e hiperhemia de los capilares de ia corteza. Esta hiperhemia explicaría los disturbios mentales y en particular la excitabilidad de las psicosis hiperfoliculínicas.

De cualquier modo que sea, cuando Lenegre y Coblentz atribuyen al hiperhormonismo en general y al hiperfoliculinismo en especial las manifestaciones cardíacas descritas; cuando Worck, a propósito de las cardiopatías en el embarazo, invoca una influencia hormónica similar en la agravación del cuadro clínico; cuando Lutembacher y Galimard, al obtener buenos resultados en ei empleo de la hormona ocitócia en manifestaciones análo_ gas, creen que el éxito se deba a una supresión o disminución en ia formación de ia hormona foliculínica, y en fin, cuando Baruk y colaboradores dan una explicación como la que hemos expuesto anteriormente, no dicen en qué forma la hormona o las hormonas pueden interferir en el determinismo del cuadro clínico.

El mecanismo a través dei cual la presencia anormal de las hormonas determina la aparición de la sintomatología extragenital descrita, no es de interpretación fácil. Las tentativas de interpretación a este respecto no son muy convincentes: ellas a lo más hacen referencia a los fenómenos finaies de la acción hormónica, dejando sin solución las diversas etapas que prece. den a estos fenómenos.

Perrault, habiendo comprobado un aumento sensible de la histamina en casos análogos, ha demostrado que el empleo de los antihistamínicos de síntesis producen un beneficio notorio. Este retorno a la intervención de la histamina como factor intermediario de la acción hormónica y al efecto antihistamínico, po_ ne el problema sobre un plano de interpretación que nosotros y otros (Herschberg. Drissche, etc.) hemos discutido ampiiamente, ya en lo que respecta a la posición que se le debe atribuir a la aminobase en la realización de la actividad hormónica, ya en jo que se refiere a la intervención del mecanismo antihistamí. nico. En ei primer caso se trataría de una acción mediadora en la que participaría el sistema nervioso; en el segundo de una 
actividad directa antagonista sobre la aminobase o indirecta sobre los procesos reguladores de la formación de la histamina. Además, teniendo presente que el poder antihistamínico no se termina dentro de estos límites (Donateili y Serafini) por cuanto se le reconoce también una modesta acción ganglioparalítica (Serafini y Genazzani), se podr'a explicar cómo algunos, en con_ diciones clínicas análogas, hemos obtenido resultados tai vez dis. cretos, usando ei Benadryl, el Fenergan, etc.

La interpretación y la documentación clínico-experimental, dada por nosotros en otro sitio sobre el empleo de los neuroplégicos en el síndromo premestrual, aclara también a grandes ras. gos ei problema actual.

En la presente casuística no es posible dudar de la participación del sistema nervioso autónomo, así como es aceptada la opinión que admite la existencia de un iazo funcional con las hormonas. Los argumentos para sustentar esta tesis son múltiples, ya en el campo experimental. ya en el clínico y no es el ca_ so de repetirlos.

Esta nueva orientación terapéutica ha tenido ya, fuera del estado gravídico, un número de interesantes contribuciones. Battermann, Grossmann y Schwimmer han obtenido resultados alentadores empleando los neuropléjicos en el síndromo anginoide; Dumas ha escrito difusamente sobre el uso de los gangiioplégicos en cardiología y ha demostrado que en las formas fun. cionales ellos deben tener una consideración especial; Petit, en fin, se ha referido a la acción benéfica de ia Clorpromazine en la taquicardia paroxística.

Los resultados obtenidos en el cuadro de las manifestaciones funcionales cardíacas durante el embarazo. le dan valor a estos éxitos y contribuyen a esclarecer la causa y el presunto mecanismo determinante de las formas que se han desencadenado en un período en el cual el más pequeño desequilibrio hormonal repercute sobre los diversos sistemas y aparatos.

Massari escribe, a propósito de sus investigaciones sobre la ansiedad y el bioqueo vegetativo: "la ansiedad constituye en la práctica clínica cuotidiana un problema urgente, lleno de sorpresivas exigencias, no colmables fácilmente. Proteger al ansioso de los "stress" uiteriores, emotivos o terapéuticos, vencer sus manifestaciones polimorfas y cambiantes, equivale a disolver, a po. ner dique a la exuberancia aberrante de su vida vegetativa y psí- 
quica". Estas palabras denuncian la necesidad de intervenir del mejor modo para alcanzar una completa rendición ail'.

No existe ninguna duda de que los simpaticolíticos, como también ios antagonistas de la acetilcolina y de la histamina, ejercen a más de una acción periférica un efecto idéntico sobre el encéfaio. Burgi ha reagrupado bajo la denominación de subs_ tancias "recepto-neurérgicas" todos aquellos fármacos que poseen una acción específica sobre los receptores periféricos aso_ ciada a una acción específica sobre el sistema nervioso central. El susodicho empleo en la terapéutica de las crisis ansiosas ha tenido comienzo desde que se observó en ios individuos sometidos a. anestesia con "cocktails", un desinterés singular en el confrontamiento del ambiente que los rodeaba sin que se verificaran modificaciones psíquicas de importancia.

Hoy se ha abandonado la idea de obtener una acción hipnótica con grandes dosis, pues sería inútil y perjudicial; como también están siendo abandonados los "cocktails", dando la preferencia absoluta (Delay y Deniker) al empleo de la Clorpromazine sola.

En el curso de este tratamiento Verdeaux y Marty encon_ traron que ias modificaciones de la circulación cerebral son notorias y caracterizadas por el adelgazamiento de los vasos y disminución de su volumen. La apariencia de tales fenómenos se estima mejor cuardo se hace una comparación con el efecto opuesto determinado por otras substancias como la foliculina, las hormonas sexuaies (esteroides). la acetilcolina y la histami_ na, con las cuales se obtiene vasodilatación acompañada de edema. Estas observaciones, confirmadas por Baruk, Launay y Lelord, son idénticas a aquellas que nosotros hemos descrito en otro campo (útero) después del tratamiento asociado de hormonas histaminérgicos y neuroplégicos.

Investigaciones experimentaies, hechas sobre animales su_ periores, han demostrado que las formaciones vegetativas del cerebro son más permeables a la Clorpromazine. de lo que lo son las céluias de conducción cerebro_espinales. De esto resulta que su acción se aplica más intensamente a nivel de las células que forman las estructuras vegetativas, las cuaies transmiten el $\mathrm{im}^{-}$ pulso nervioso a las células efectoras del sistema neuro-vegeta. tivo y al sincitio nervioso distal que se encuentra bajo ia dependencia ya del simpático ya del parasimpático. 
Fara Delay la Clorpromazine obraría poniendo en reposo el sistema nervioso vegetativo periférico y central e interrumpiendo o atenuando ios procesos reactivos que en muchos casos constituyen el substrato de la enfermedad. Más interesante es la in. terpretación dada por Belloni. Este sostiene que el preparado obra en sitio central bloqueando el sistema reticular ascendente, ei que, según Moruzzi, constituiria "el sistema de amplificación puesto entre la periferie sensitiva y el cerebro”. Tal bloqueo provocaría una "deaferenciación" dei sistema reticular hecho más rócil por la acción periférica de la Clorpromazine. Esta capacidad de bloqueo buscaría interrumpir asi la acción hormónica primitiva.

De cualquier modo que sea, como también lo ha dicho recientemente Burgi, con el empleo de ios neuroplégicos se ha llegado a una nueva orientación terapéutica de las distonias neuro_vegetativas de sentido erético. Esta limitación es fundamental para no crear confusión con las manifestaciones de insufi_ ciencia que deben ser lógicamente tratadas en otra forma.

El uso de estos fármacos. calmando la hiperactividad neuro_vegetativa, crea un estado de indiferencia y de desinterés hacia ei ambiente en el cual vive la paciente, removiendo as el componente ansioso y la espina irritativa.

La forma de empleo merece unas palabras. Baruk, Launey y Lelord en los casos de crisis ansiosas han suministrado la Clorpromazine a la dosis de 2 comprimidos de 25 miligramos por ios dos primeros días; una cápsuia de 50 miligramos y un comprimido de 25 miligramos el tercer día; y dos inyecciones al día de 50 miligramos a partir del cuarto día. La experiencia nuestra nos aconseja usar prevalentemente la vía oral; la parenteral está reservada soio a aquellos casos de cierta gravedad; por otra parte, razones evidentes de practicabilidad hacen preferir la va oral. En cuanto a la dosis diaria es difícil y complejo formular un esquema preciso; es preferible regular la dosis de acuerdo con ei sujeto tratado. De todos modos, en los casos referidos se ha seguido un ritmo progresivamente creciente para de'spués dis_ minuir hacia el fin de ia cura. Las interrupciones bruscas deben evitarse en todo caso. En lo que respecta a la tolerancia es prudente observar estos pacientes para evitar inconvenientes eventuales (lipotimias, hipotensión taquicardia, hipotermia, etc.), que en los casos de que tratamos no se han presentado. En las crisis ansiosas es aconsejable iniciar con comprimidos de 25 
miligramos y no pasar después de los primeros dos días de más de 50 miligramos por dia; para luego decrecer desde el décimo día.

El período progresivo de la cura no pasa de veinte días. En las disfunciones cardíacas se puede iniciar con 50 miligramos por día, pero sin superar los 75 miligramos; la duración dei tratamiento puede ser más breve.

Para concluir: en los dos tipos de manifestaciones conside_ radas, durante el embarazo, el empleo de la Ciorpromazine resuel. ve un dilema terapéutico que especialmente en estos últimos tiempos ha venido presentándose, ya por la mayor frecuencia con que tales síndromos se manifiestan. ya por ia dificultad encontrada en la escogencia de una guía terapéutica de eficacia segura.

\section{BIBLIOGRAFIA}

BARUK. LAUNAY e LELORD.-Annales Médico Psychol, 2, 66. 1954.

BARUK.-Précis de Psychiatrie. Niasson Ed. 441. 1950.

BARUK, DAVID, RACINE, VALANCIEN.-Revue neurologique 80, 487. 1948.

BARUK, DAVID, RACINE, VALANCIEN e BORENSTEIN.- Amnales médico-psychologique. 2. 1947.

BARUK e RACINE.-Annales médicc-psychologiques. 1946.

CAHN, DUBRASQUET e MELON.-Anesthésie et Anelgésie 10, febraio. 1953.

CAZZOLA.-Ganglioplegici e anticolinergici in Obstetricia e Ginecologia. Ed. Salpietra. Firenze. 1955.

DELAY, DENIKER, HARL.-Annales médico-psychologiques 2, 267. 1952. DUMAS.-Concours Medical 23, 2.273. 1954.

DONATELLI e SERAFINI.-Gli antistaminici di sintesi. E. S. I. Ed. Napoli. 1951.

DRIESSCHE e FAUOZSKI.-Bruseles Médñ 30, 957. 1950.

CAMMON e CHURCHILL.-Am. J. Set. 217, 143. 1949.

GENAZZANI e DONATELLI.-Arch. Int. Pharm, et. Therap. 88, 209. 1951.

JACOBSON.-Progressive relaxation.-Univ. Chicago Press. 1947.

HUNTER e WATERFALL.-Lancet 336. 1948.

SIGWALD e BUOTTIER.-Presse Medical 29. 1953.

STAEHELIN e KIELHOLZ.-Smhewis. Ned. Wachr. 83, 581. 1953.

HERSCHNERG.-Gyn. Prat. 3. 263 e 315. 1952.

MASSARI.-Minerva Medica 44, 231. 1953.

LUTENBACHER e GALIMARD.-Presse Medical 73, 885. 1948.

WERKO.-Acta Obstet. et Gynec. Scandinavica 33, 162. 1954.

BURGI.-Médicine et Hygiene 289, 129. 1955.

LENEGRE e COBLENTZ.-Cit. da LUTEMBACHER e GALIMARD. 